\title{
ICONO14
}

\section{Youtubers: identificación y motivos de agrado de la audiencia}

\section{Youtubers: identification and reasons for liking of the audience}

\section{Youtubers: identificação e razões para gostar da audiencia}

Oihane Korres Alonso ${ }^{1}$ (iD

Iciar Elexpuru-Albizuri² ${ }^{2}$

${ }^{1}$ Personal Docente e Investigador Facultad de Educación y Deporte. Departamento de Educación. Universidad de Deusto, España

${ }^{2}$ Personal Docente e Investigador Facultad de Educación y Deporte. Departamento de Educación. Universidad de Deusto, España

Recibido: 24/06/2021; Revisado: 27/07/2021; Aceptado: 26/10/2021; Publicado: 01/01/2022

Para citar este artículo: Korres Alonso, O. y Elexpuru Albizuri, I (2022). Youtubers: identificación y motivos de agrado de la audiencia Icono 14, 20(1). https://doi.org/10.7195/ri14.v20i1.1761

\section{Resumen}

Actualmente, los youtubers se han convertido en modelos a seguir, especialmente, para los más jóvenes. En este artículo se analizan las interacciones de los seguidores de los youtubers españoles de éxito en términos de comentario y tema, identificación y motivos de agrado. Para ello, se analizan los comentarios de la audiencia a los vídeos de 10 youtuber de éxito españoles, seleccionados a partir de un estudio exploratorio atendiendo a los siguientes criterios: influencers presentes en YouTube e Instagram, edad (18-30 años), orientación sexual, notoriedad (entre 200.000 y 34 millones en el canal de YouTube) y variedad temática de su canal. Mediante el análisis de contenido de 1000 comentarios, se reconocen los argumentos y aspectos comunes que señalan los 
seguidores. Los resultados muestran, por un lado, que una categoría relevante es la identificación con los youtubers, destacando la identificación aspiracional y la similitud con el influencer, y, por otro lado, que los seguidores valoran, especialmente, el humor y la personalidad del youtuber, seguido de su atractivo físico, y, en menor medida, de su inteligencia y riqueza/éxito. Se subraya el interés de seguir ahondando en la investigación sobre el impacto de los influencers en la formación de los más

jóvenes, tanto explorando los mensajes y representaciones que transmiten, como su recepción e interpretación.

Palabras clave: Youtuber; influencers; seguidores; identificación; motivos de agrado; análisis de contenido

\section{Abstract}

Nowadays, youtubers have become role models, especially for young people. This article examines the interactions of the followers of successful Spanish youtubers in terms of comment and topic, identification, and reasons for liking them. For this purpose, audience comments on the videos of 10 successful Spanish youtubers were analysed, selected from an exploratory study based on the following criteria: influencers active on YouTube and Instagram, age (18-30 years), sexual orientation, notoriety (between 200,000 and 34 million on the YouTube channel) and thematic variety of their channel. Through content analysis of 1000 comments, the arguments and common elements provided by the followers have been identified. The results show, on one hand, that a relevant category is identification with youtubers, expressly, aspirational identification and similarity with influencers, and, on the other hand, that followers value, especially, the humour and personality of the youtuber, followed by the physical attractiveness, and, to a lesser extent, the intelligence and wealth/success. The interest in further research into the impact of influencers on the education of young people is underlined, both through the analysis of the messages and representations transmitted, as well as their reception and interpretation.

Keywords: Youtuber; influencers; followers; identification; reasons for liking; content analysis

\section{Resumo}

Actualmente, os youtubers tornaram-se modelos a seguir, especialmente para os jovens. Neste artigo analisamos as interacções dos seguidores de youtubers espanhóis de sucesso em termos de comentários e tema, identificação e razões para gostar. Para tal, analisamos os comentários do público aos vídeos de 10 youtubers espanhóis de sucesso, seleccionados a partir de um estudo exploratório baseado nos seguintes critérios: influenciadores presentes no YouTube e Instagram, idade (18-30 anos), orientação sexual, notoriedade (entre 200.000 e 34 milhões no canal YouTube) e variedade temática do seu canal. Através da análise do conteúdo de 1000 comentários, são identificados os argumentos e elementos comuns fornecidos pelos seguidores. Os resultados mostram, por um lado, que a identificação com os youtubers emerge como uma categoria importante, destacando a identificação aspiracional e semelhança com os influenciadores, e, por outro lado, que os seguidores valorizam, especialmente, o humor e a personalidade do youtuber, seguidos pela sua atracção física, e, em menor grau, a sua inteligência e riqueza/sucesso. Sublinhamos o interesse de mais investigação sobre o impacto dos influenciadores na educação dos jovens, tanto através da análise dos seus conteúdos e representações, como da sua recepção e interpretação. 
Palavras-chave: Youtuber; influencers; seguidores; identificação; razões para gostar; análise de conteúdo

\section{Introducción}

El desarrollo de las nuevas tecnologías de la información ha traído consigo la creación y proliferación de las redes sociales virtuales, definidas como comunidades que, a través de Internet, ponen en contacto a personas que interactúan entre sí, compartiendo información e intereses (Morduchowicz, Marcon, Sylvestre y Ballestrini, 2010), modificando, así, la forma de ser, estar y relacionarse con los demás. Como señalan Vizcaíno-Verdú, De Casas-Moreno y Aguaded (2019), los usuarios, por medio de experiencias diarias en la Red, comparten sus ideas e inquietudes, convirtiendo la cultura participativa en una vía de colaboración y transformando las redes sociales en "un instrumento de difusión, de promoción, de modificación de comportamientos sociales, de definición de identidades, de agrupación y movimiento social, entre otras temáticas" (p.217). Así, los social media se establecen como espacios privilegiados para la interrelación social en el proceso de construcción de la identidad (Buckingham, 2008; Cover, 2012; Aran-Ramspott, Fedele y Tarragó, 2018; Regueira, Alonso-Ferreiro y Da-Vila, 2020).

Como subrayan distintos estudios (AIMC, 2018; Kádeková y Holiencinová, 2018; Eurostat, 2020), las redes sociales se han convertido en indispensables en el día a día, especialmente, para los más jóvenes. En estas redes emergen y se consolidan nuevos referentes mediáticos, denominados influencers. En este sentido, De Veirman, Cauberghe y Hudders (2016, p.1) definen a los influencers como "personas que construyen una gran red de seguidores y son consideradas como creadoras de tendencias de confianza" en uno o varios ámbitos. En concreto, en España, el $85 \%$ de los jóvenes entre 16 y 30 años utiliza las redes sociales para seguir a influencers (IAB Spain, 2018), quiénes pueden repercutir en el pensamiento y comportamiento de sus seguidores. Kádeková y Holiencinová (2018) subrayan cuatro tipos de influencers, considerando los contenidos que generan y las plataformas que utilizan: el blogger, el youtuber o vlogger, el celebrity y el instagrammer.

En este contexto, el estudio que se presenta se centra en el segundo tipo de influencers, es decir, en los youtubers, dada la relevancia que han adquirido en los últimos años. Chad Hurley, Steve Chen y Jawed Karim fundaron la red social YouTube en 2005 con el fin de compartir vídeos online y, actualmente, tiene más de dos mil millones de usuarios (lo que representa a una tercera parte de los consumidores de Internet), destacando los que tienen entre 18 y 34 años, y está disponible en 80 idiomas (YouTube, 2021). Así, YouTube está reemplazando al medio televisivo entre los jóvenes, por su contenido interactivo y dirigido a la audiencia, que es genuino y crea comunidades (Tolbert y Drogos, 2019). 
Como red social, YouTube permite a los usuarios convertirse en creadores y difusores de contenidos audiovisuales, es decir, en youtubers o vloggers (Vizcaíno-Verdú et al., 2019). Sin embargo, no todos los usuarios de YouTube que generan y difunden contenidos en la plataforma alcanzan el mismo volumen de seguidores e influencia. En este sentido, Bonaga y Turiel (2016, pp. 120-121), definen al youtuber como:

Todo aquel individuo que, con una periodicidad regular y constante, sube contenidos a la plataforma y consigue convertir en retribución monetaria el número de visualizaciones de un archivo dado. Lo que se conoce con el anglicismo de 'monetizar las visitas'. De esta manera sólo tendrían el crédito de "creador" las personas que han conseguido hacer de YouTube no ya una afición, sino su profesión. Y lo más relevante de los youtubers de éxito, de lo que hemos denominado "creadores", es su cualidad de llegar a ser influencers.

Siguiendo a Regueira et al. (2020), los youtubers son, generalmente, jóvenes capaces de crear grandes masas de seguidores que se sostienen en el tiempo, generando, así, una comunidad que gira en torno a su figura y define símbolos identitarios. En esta línea, Rego y Romero-Rodríguez (2016, p. 198) señalan que los youtubers, en su mayoría, son "jóvenes que tienen pocos años más que el target que les sigue, y tratan temas de actualidad de una forma coloquial, convirtiéndose, en cierta medida, en líderes de opinión".

Así, el estudio en torno a YouTube y los youtubers se ha convertido en un área de interés para la investigación (Vizcaíno-Verdú et al., 2019). En el contexto español, se pueden encontrar, entre otros, estudios sobre el modelo de negocio de Youtube (Larrañaga y Ruiz, 2009), la discursividad y el lenguaje de los youtubers (Rego y Romero-Rodríguez, 2016; Sabich y Steinberg, 2017), el contenido que transmiten en sus vídeos los principales youtubers seguidos por niños (Renés, Gozálvez y Berlanga, 2020), el nuevo perfil profesional de los influencers, por ejemplo, mediante el análisis del caso de Verdeliss (Elorriaga y Monge, 2018), o, desde una perspectiva de género, la participación y representación de la mujer en esta red social (Regueira et al., 2020) o las diferencias de género en cuanto a consumo, creación y difusión de contenidos (Fernández-de-Arroyabe-Olaortua, LazkanoArrillaga y Eguskiza-Sesumaga, 2018). Sin embargo, aún es escasa la investigación sobre el impacto de los influencers y, concretamente, de los youtubers (Rego y Romero-Rodríguez, 2016; Aran-Ramspott et al., 2018; Elorriaga y Monge, 2018; Tolbert y Drogos, 2019), así como de la recepción e interacción de su audiencia.

Por tanto, en contexto mediático actual resulta relevante indagar en el impacto de los nuevos medios y sus referentes, como son YouTube y los youtubers, en los más jóvenes. Existe una amplia investigación sobre los medios de comunicación tradicionales y su influencia, donde diversos estudios han puesto de relieve la capacidad socializadora del medio televisivo, especialmente, de la ficción televisiva y sus personajes, que pueden resultar cercanos, ser comparados por la audiencia, e influir en la configuración de sus valores o actitudes (Livingstone, 1998; Hoffner y Buchanan, 2005; Igartua, 2010; entre otros). En este sentido, la identificación con el personaje, definida como un proceso 
cognitivo y emocional a través del cual el espectador se pone en el lugar del protagonista y toma su perspectiva (Cohen, 2001), resulta fundamental a la hora de entender los efectos del medio (Cohen, 2001; Eyal y Rubin, 2003; Hoffner y Buchanan, 2005; Moyer-Gusé, 2008; Korres y Elexpuru, 2016). Así, la identificación con los personajes se compone de distintas dimensiones: por un lado, destaca la relación empática (emocional o cognitiva) que se establece con los personajes (Cohen, 2001; Eyal y Rubin, 2003; Moyer-Gusé, 2008; Igartua, 2010); por otro lado, resulta relevante la similitud percibida con los mismos (Eyal y Rubin, 2003; Hoffner y Buchanan, 2005; Moyer-Gusé, 2008; Korres y Elexpuru, 2016); además, se subraya el deseo de parecerse al personaje (Cohen, 2001; Hoffner y Buchanan, 2005; Moyer-Gusé, 2008; Korres y Elexpuru, 2016); y, por último, se encuentra la interacción parasocial (Livingstone, 1998; Eyal y Rubin, 2003; Korres y Elexpuru, 2016), que se refiere al proceso a través del cual el espectador mantiene una pseudorelación con el personaje (Moyer-Gusé, 2008).

Del mismo modo que los referentes televisivos, actualmente, los youtubers se convierten en líderes de opinión y modelos a seguir por los jóvenes (Fernández-de-Arroyabe-Olaortua et al., 2018). Así, pueden actuar como modelos de referencia, también a través de mecanismos de identificación y empatía (Aran-Ramspott et al., 2018), ya que son "personas cercanas y accesibles, que desafían los márgenes de la intimidad y la privacidad" (Regueira et al., 2020, p. 63). En este sentido, los jóvenes de hoy consideran más identificables a los youtubers, de quienes valoran que tengan carácter y sean auténticos, que a las celebridades tradicionales (Defy Media, 2016). Como señalan Tolbert y Drogos (2019), comparado con los personajes fantásticos de ficción de los medios tradicionales, el hecho de que los youtubers existan en la realidad y comenzaran como personas "normales" produciendo vídeos en sus casas antes de convertirse en celebridades, facilita la vinculación de la audiencia. Dicho estudio también refleja, por un lado, que los jóvenes quieren parecerse a sus youtubers favoritos, de quienes valoran que sean inteligentes y divertidos, y que este deseo aspiracional se relaciona con la similitud percibida, $y$, por otro lado, que establecen relaciones parasociales con los youtubers, experimentando sentimientos de amistad.

Los youtubers construyen su marca personal mediante la exposición de sus vidas personales y la proximidad hacia sus seguidores, a través de una comunicación directa y creativa, mostrando autenticidad y cercanía a la audiencia, y recibiendo el agradecimiento de sus followers por "haberles sacado una sonrisa» con sus vídeos (Bonaga y Turiel, 2016). Así, como señalan Fernández-de-Arroyabe-Olaortua et al. (2018), en Internet los adolescentes consumen, crean y difunden contenidos relacionados con el ocio. En esta línea, Aran-Ramspott et al. (2018), encuentran que a los preadolescentes les atraen los youtubers, principalmente, por el humor y la proximidad a sus propios intereses. Como motivos de atracción también destacan elementos como la coolness, la identificación con algún youtuber y poder compartirlo con sus iguales. Además, admiran "su vis cómica y sus conocimientos más que su aspecto físico o la imagen de marca que puedan representar" ( $p$. 57). Este estudio también indica que desconfían de la fama y los riesgos que pueda conllevar el trabajo de los youtubers, y que se muestran críticos ante las actitudes ofensivas y discriminatorias. En esta línea, en relación con los personajes televisivos, diversos 
estudios muestran que los adolescentes valoran su personalidad $y$, principalmente, su simpatía y humor (Medrano, Cortés, Aierbe y Orejudo, 2010; Medrano y Martínez de Morentín, 2012; Bermejo, 2012; Korres y Elexpuru, 2016). También valoran del personaje sus relaciones sociales, $y$, en menor medida, su inteligencia, atractivo físico, riqueza/ éxito y trabajo (Korres y Elexpuru, 2016). En cuanto al atractivo físico del personaje, algunos estudios destacan que es un elemento importante (Ruiz, Conde y Torres, 2005), sin embargo, otros autores indican que no es un aspecto relevante (Medrano et al., 2010; Medrano y Martínez de Morentín, 2012).

Como señalan Scolari y Fraticelli (2016), el éxito de los youtubers parece sostenerse en el contacto individualizado que permite el dispositivo, ya que además de generar un sujeto que produce videos, "genera un espectador activo que deja su marca en la interfaz y participa activamente haciendo comentarios, señalando lo que le gusta y no le gusta, suscribiéndose a uno u otro canal y contactándose con el youtuber por medio de otros hipermedios como Twitter" (p. 1689). Por tanto, es relevante indagar los mensajes que se producen en los medios, ya que la audiencia también interactúa a través de estos (De la Fuente, Lacasa y Martínez-Borda, 2019). Así, alrededor de la figura del youtuber se crea una comunidad con símbolos identitarios, en la que la interacción es "bidireccional, las y los youtubers interpelan a su audiencia, quien, a su vez, le devuelve feedback" (Regueira et al., 2020, p. 63).

En este sentido, este estudio explora los comentarios de la audiencia a los videos en YouTube, para ahondar en la relación que establecen con los youtubers. A través de este conocimiento se podrá favorecer el desarrollo de propuestas orientadas a promover la alfabetización mediática. Como señalan diversos autores, se hace cada vez más necesario contribuir en el desarrollo de competencias mediáticas, especialmente, en aquella relacionada con la dimensión de ideología y valores (Díaz-Aguado y Falcón, 2013). En su investigación, De la Fuente, Lacasa y Martínez-Borda (2019) indican que, Internet y las redes sociales constituyen un importante contexto no formal para el aprendizaje, formando un espacio donde se crean nuevas prácticas y discursos digitales, combinando entornos reales y virtuales, y creando comunidades en las que cualquier persona puede participar. De este modo, resulta necesario continuar investigando sobre estos nuevos medios y sus referentes, así como desarrollar propuestas educativas que favorezcan una mirada crítica ante los mismos. En este sentido, Renés, Gozálvez y Berlanga (2020), siguiendo estudios previos como el de Escobar-Román (2011), Callejo y Gutiérrez (2014) o Morgan y Kristjánsson (2017), ofrecen algunas recomendaciones para favorecer el uso adecuado de las redes sociales entre los más jóvenes, por ejemplo, promover una lectura reflexiva y crítica sobre los discursos de los youtubers, elaborar recursos didácticos para analizar este tipo de plataformas en los centros educativos, o capacitar a los docentes y a las familias en la educación digital. 


\section{Material y métodos}

Este estudio forma parte de una investigación más amplia denominada "Construcción de las identidades de género, valores y estereotipos juveniles en los social media: I@s influencers y las audiencias Millennial y Centennial", financiado por el Centro Reina Sofía (convocatoria 2018) ${ }^{1}$. Esta investigación se ha desarrollado en tres fases:

1) Un análisis exploratorio cuantitativo y uno cualitativo de 52 perfiles de influencers españoles populares (y 156 vídeos de YouTube);

2) Un análisis en profundidad de 10 influencers españoles ( 5 de sexo femenino y 5 de sexo masculino) escogidos de los 52 perfiles del análisis exploratorio;

3) Un análisis de la audiencia llevado a cabo mediante análisis de contenido de 1000 comentarios de seguidores en YouTube. Para cada uno de los 10 vídeos de YouTube analizados en la fase 2 , se han analizado los 100 comentarios con más impacto. Entre los aspectos analizados se encuentran: comentario y tema; identificación con el youtuber; motivos de agrado y valores percibidos.

Los resultados derivados de las fases 1 y 2 , que se centran, principalmente, en el análisis de los perfiles y vídeos de influencers españoles de éxito, se encuentran en proceso de publicación (Fedele, Aran-Ramspott, Elexpuru y Korres, en prensa). En concreto, en este artículo se presentan los resultados y conclusiones de la fase 3. El objetivo es analizar los comentarios e interacciones de los seguidores a los vídeos de 10 youtubers españoles de éxito en términos de comentario y tema, identificación y motivos de agrado. Mediante el análisis de contenido se identifican los argumentos y los aspectos comunes que aportan los seguidores en sus comentarios.

\subsection{Selección de la muestra}

Para la selección de los y las youtubers españoles/as sobre los que analizar los comentarios de sus seguidores, se ha buscado la mayor diversidad posible entre los perfiles, atendiendo a los siguientes criterios: influencers presentes en YouTube e Instagram, edad (18-30 años), orientación sexual, notoriedad (entre 200.000 y 34 millones en el canal de YouTube) y variedad temática de su canal. En este sentido, en cuanto a la popularidad, el criterio se ha adaptado a los perfiles de influencers masculinos y femeninos, contando los primeros entre 1 y 34 millones de seguidores y la de las segundas entre los 200.000 y los 2,5 millones. En cuanto a la diversidad temática, pese a que la temática mayoritaria en el caso de los hombres parece ser más homogénea, cabe señalar que existe cierta diversidad bajo la etiqueta "entretenimiento". No obstante, debido, posiblemente, al criterio de notoriedad, la muestra no ha podido tener en cuenta la variedad racial, ni ha podido incluir canales especializados en temática LGBTIQ+. Finalmente, se han tenido que excluir canales que fueran exclusivamente de gamers, ya que sus videos no ofrecían material en línea con los objetivos del estudio. 
A partir de las estadísticas correspondientes al periodo de análisis (2018-2019) llevado a cabo en un estudio exploratorio, se han seleccionado los 5 perfiles masculinos y los 5 perfiles femeninos que garantizaran la mayor variedad posible en la muestra. Los youtuber masculinos seleccionados son los de El Rubius, AuronPlay, Wismichu, Celopan y Jaime Altozano. Los femeninos, a su vez, son los de Dulceida, Yellow Mellow, Paula Gonu, Herrejón y La gata de Schrödinger.

A la hora de seleccionar el vídeo de cada youtuber sobre el que se analizan los comentarios de los seguidores (tabla 1), se han considerado criterios temporales publicados entre enero de 2018 y septiembre de 2019-, y temáticos -vídeos del género entrevistas y/o blogs, según la clasificación establecida por Scolari y Fraticelli (2016), puesto que en ambos casos los influencers se exponen a nivel personal, ya sea respondiendo a las preguntas de la audiencia, o manifestando su opinión sobre un tema concreto-. 
Tabla 1. Videos de los youtubers sobre los que se analizan los comentarios

\begin{tabular}{|c|c|c|}
\hline Youtuber & Título del vídeo & Resumen \\
\hline El Rubius & $\begin{array}{l}50 \text { COSAS SOBRE Mí } 2019 \text { by } \\
\underline{\text { Rubius }}\end{array}$ & $\begin{array}{l}\text { El youtuber responde preguntas } \\
\text { cortas y personales de la } \\
\text { audiencia tales como cuál es su } \\
\text { comida o su deporte favorito }\end{array}$ \\
\hline Auronplay & $\begin{array}{l}\text { CÓMO LIGAR (en la } \\
\text { Prehistoria) }\end{array}$ & $\begin{array}{l}\text { El youtuber ironiza sobre un vídeo } \\
\text { que ha encontrado en la red sobre } \\
\text { cómo ligar }\end{array}$ \\
\hline Wismichu & $\begin{array}{l}\text { Respondiendo a vuestras } \\
\text { preguntas por la calle }\end{array}$ & $\begin{array}{l}\text { El youtuber responde a las } \\
\text { preguntas de sus suscriptores, } \\
\text { como si es vegano o las series } \\
\text { que le marcaron en la infancia }\end{array}$ \\
\hline Jaime Altozano & $\begin{array}{l}\text { Todo sobre mí -Especial } 1 \\
\text { Millón de Suscriptores / Jaime } \\
\underline{\text { Altozano }}\end{array}$ & $\begin{array}{l}\text { El youtuber responde a las } \\
\text { preguntas de la audiencia tales } \\
\text { como cuánto gana o si quiere } \\
\text { tener hijos }\end{array}$ \\
\hline Celopan & $\begin{array}{ll}\text { PREGUNTAS QUE } & \text { HE } \\
\text { EVADIDO - Celopan } & \end{array}$ & $\begin{array}{l}\text { El youtuber responde preguntas } \\
\text { personales planteadas por la } \\
\text { audiencia tales como si querría } \\
\text { casarse o cómo conoció a su } \\
\text { pareja }\end{array}$ \\
\hline Dulceida & $\begin{array}{l}\text { TAG DEL } \quad \text { YOUTUBE } \\
\underline{\text { HIPÓCRITA - DULCEIDA }}\end{array}$ & $\begin{array}{l}\text { TAG en que la youtuber responde } \\
\text { preguntas relativas al universo } \\
\text { YouTube, como a quién envidia o } \\
\text { admira. }\end{array}$ \\
\hline Yellow Mellow & 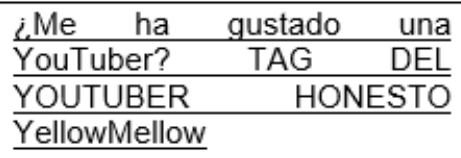 & $\begin{array}{l}\text { TAG en que la youtuber responde } \\
\text { preguntas relativas al universo } \\
\text { YouTube, como a quién envidia o } \\
\text { admira. }\end{array}$ \\
\hline Paula Gonu & $\begin{array}{l}\text { REACCIONANDO A LO QUE } \\
\text { SUPONÉIS SOBRE MI / } \\
\text { @.paulagonu }\end{array}$ & $\begin{array}{l}\text { TAG en el que la youtuber } \\
\text { confirma o desmiente a las } \\
\text { suposiciones de la audiencia y } \\
\text { aporta información personal }\end{array}$ \\
\hline Herrejón & $\begin{array}{l}\text { LO QUE SUPONÉIS SOBRE } \\
\text { MI (¿SOY } \\
\text { BISEXUDEL } \\
\text { HERREJÓ), }\end{array}$ & $\begin{array}{l}\text { TAG en el que la youtuber } \\
\text { confirma o desmiente las } \\
\text { suposiciones de la audiencia y } \\
\text { aporta información personal }\end{array}$ \\
\hline $\begin{array}{l}\text { La Gata } \\
\text { Schrödinger }\end{array}$ & $\begin{array}{l}\text { ¿Cómo llevo ser YouTuber? } \\
\text { ¿Trabajo para la } \text { ClA? } \\
\text { PREGUNTAS } \\
\text { RESPUESTAS }\end{array}$ & $\begin{array}{l}\text { La youtuber responde preguntas } \\
\text { planteadas por la audiencia tales } \\
\text { como cuál es su grupo musical } \\
\text { favorito }\end{array}$ \\
\hline
\end{tabular}

Fuente: Elaboración propia

En cuanto al análisis de los comentarios e interacciones de los seguidores, se han analizado los 100 comentarios con más impacto (número de reacciones) en los 10 vídeos 
seleccionados (un vídeo de cada youtuber). Es decir, se han indagado un total de 1000 comentarios.

\subsection{Procedimiento de recogida y análisis de datos}

Los comentarios se han recogido durante los meses de enero y febrero de 2020 y se han transcrito de manera literal. Estos comentarios se han analizado mediante la técnica de análisis de contenido con el software Atlas.Ti, con el fin de identificar y categorizar aquellos comentarios que son relevantes para la investigación. Como punto de partida se han utilizado las categorías recogidas en estudios previos relacionados con personajes de ficción televisiva (Medrano et al., 2010; Bermejo, 2012; Korres y Elexpuru, 2016), revisándolas y adaptándolas posteriormente al contexto de los youtubers. En primer lugar, se definieron las categorías emergentes en el análisis de los comentarios, que, atendiendo al criterio teórico, se depuraron en sucesivas lecturas. Posteriormente, estas categorías se aglutinaron en núcleos de análisis significativos, considerando que conservaran una esencia común y se ajustaran al contenido de la categoría. Así, se han analizado en estos comentarios los siguientes aspectos: comentario y tema; identificación con el youtuber y motivos de agrado reflejados.

Las categorías de respuesta con un bajo porcentaje no se incluyen en este trabajo, para poner el foco sobre los argumentos que más señalan los seguidores en sus comentarios (tabla 2). 
Tabla 2. Categorías sobre los youtubers

\begin{tabular}{|c|c|c|}
\hline Categoría & Subcategoria & Definición \\
\hline \multirow[t]{4}{*}{$\begin{array}{l}\text { Tema } \\
\text { posición }\end{array}$} & $\begin{array}{l}\text { Refiere al tema o al } \\
\text { youtuber }\end{array}$ & $\begin{array}{l}\text { El autor/a del comentario hace referencia al tema o al } \\
\text { youtuber. }\end{array}$ \\
\hline & A favor/ En contra & $\begin{array}{l}\text { El autor/a del comentario se posiciona a favor o en } \\
\text { contra del tema o el/la youtuber. Se considera a favor } \\
\text { todo comentario que no se posiciona en contra. }\end{array}$ \\
\hline & $\begin{array}{lr}\text { Enlaza a } & \text { otros } \\
\text { contenidos } & \text { de } \\
\text { Internet } & \end{array}$ & $\begin{array}{l}\text { El autor/a del comentario hace referencia a otros } \\
\text { contenidos de la red. }\end{array}$ \\
\hline & $\begin{array}{l}\text { Contiene emoticonos } \\
\text { y/o emojis }\end{array}$ & $\begin{array}{l}\text { El autor/a del comentario utiliza emoticonos en su } \\
\text { mensaje. }\end{array}$ \\
\hline \multirow{4}{*}{$\begin{array}{l}\text { Identificación } \\
\text { con el } \\
\text { youtuber }\end{array}$} & Similitud & $\begin{array}{l}\text { El autor/a del comentario se reconoce en el youtuber } \\
\text { o en sus experiencias vitales. }\end{array}$ \\
\hline & $\begin{array}{l}\text { Interacción } \\
\text { parasocial }\end{array}$ & $\begin{array}{l}\text { El autor/a del comentario interactúa con el youtuber } \\
\text { del mismo modo que si lo conociese en persona. Es } \\
\text { decir, habla al youtuber directamente, de tú a tú. }\end{array}$ \\
\hline & Aspiracional & $\begin{array}{l}\text { El autor/a del comentario indica que le gustaría } \\
\text { parecerse o ser el youtuber. }\end{array}$ \\
\hline & $\begin{array}{l}\text { Empatía con el } \\
\text { youtuber }\end{array}$ & $\begin{array}{l}\text { El autor/a del comentario se implica con el youtuber, } \\
\text { poniéndose en su piel y mostrando preocupación por } \\
\text { su futuro. }\end{array}$ \\
\hline \multirow[t]{5}{*}{$\begin{array}{l}\text { Motivos } \\
\text { agrado }\end{array}$} & Humor/Diversión & $\begin{array}{l}\text { El autor/a del comentario señala que el youtuber le } \\
\text { hace gracia y le resulta divertido. Se han interpretado } \\
\text { los emoticonos o "jajaja", dentro del contexto, como } \\
\text { humor y diversión. }\end{array}$ \\
\hline & Riqueza/Éxito & $\begin{array}{l}\text { El autor/a del comentario subraya el éxito personal o } \\
\text { profesional, la riqueza y/o los bienes materiales del } \\
\text { youtuber. }\end{array}$ \\
\hline & Inteligencia & $\begin{array}{l}\text { El autor/a del comentario destaca que le gusta que el } \\
\text { youtuber sea inteligente y que utilice sus } \\
\text { conocimientos de la mejor manera posible. }\end{array}$ \\
\hline & Atractivo físico & $\begin{array}{l}\text { El autor/a del comentario subraya que le gusta el } \\
\text { youtuber por su físico o su apariencia exterior. }\end{array}$ \\
\hline & Personalidad & $\begin{array}{l}\text { El autor/a del comentario alaba un rasgo de la } \\
\text { persona del youtuber. }\end{array}$ \\
\hline
\end{tabular}

Fuente: Elaboración propia

\section{Resultados}

A continuación, se exponen los resultados obtenidos en este estudio (los comentarios de los followers se transcriben de manera literal). 


\subsection{Tema y posición}

En cuanto al tema y la posición de los comentarios estudiados, se encuentra que:

1)Refiere al tema o al youtuber: los comentarios analizados se refieren, mayoritariamente, al tema del vídeo o al youtuber que lo realiza ( $n=962,96,2 \%)$. Por ejemplo:

"THIS IS THE MOMENT; TONIGHT IS THE NIGHT. Así me pongo a gritar cada vez que subes vídeo Melo \#\#" (Comentario al vídeo de YellowMellow).

2)A favor/En contra: la mayoría de los comentarios se muestran a favor del contenido del vídeo o del youtuber que lo realiza $(n=945,94,5 \%)$, mientras que un menor número se muestra en contra ( $n=55,5,5 \%)$. Por ejemplo:

A favor: "Esperaba este vídeo con ansias, y me ha encantado! Mis miércoles son mejores con tus vídeos\#\#" (Comentario al vídeo de Dulceida).

En contra: "Chica, te enrollas tanto que al final dijiste 5 cosas que la gente supone, $y$ seguro que te mandaron cientos de ellas... en fin! Yo ya no sé para que sigo viendo tus videos si ya no me aportan lo más mínimo" (Comentario al vídeo de Paula Gonu).

3)Enlaza a otros contenidos de Internet: algunos usuarios hacen referencia a otros contenidos de la red en sus comentarios, especialmente, a otros youtubers o influencers ( $n=117,11,7 \%)$. Por ejemplo:

"Te conocí por Calle y poché hace un tiempo y me re encantas.\#" (Comentario al vídeo de Dulceida).

4)Contiene emoticonos y/o emojis: otro aspecto destacado es el uso de emoticonos en los comentarios ( $\mathrm{n}=582,58,2 \%)$. Por ejemplo:

"Ayyy Celo eres precioso me encantas \# 1pd: yo dama de honor de la boda con coso (obviosuly\#\#\#)\#\# 2Pd: amé el saludo del final\#\#\#\#" (Comentario al vídeo de Celopan).

\subsection{Identificación con el youtuber}

La identificación con los youtubers se erige como una categoría relevante en los comentarios de sus seguidores. De los 1000 comentarios, 535 hacen referencia a la identificación con el youtuber. Además, se observa que este proceso de identificación puede darse por distintas razones:

1) Interacción parasocial: destaca que los seguidores se relacionan con el youtuber como si fuese alguien cercano, hablándole de tú a tú $(n=467,46,7 \%)$. Por ejemplo:

"Una pregunta para cuando puedas la respondes. Que pasaria si fueras alguien mas, un ejemplo, tu seas yo, una persona comun y corriente, alejado de todo lo que es Rubius???" (Comentario al vídeo de El Rubius). 
Cabe señalar que se han identificado unos rasgos que permiten concretar cómo se da la interacción parasocial, entre ellos, destacan:

- Felicita trabajo ( $n=94,9,4 \%)$ : El seguidor valora la profesión desempeñada o el video realizado por el youtuber. Por ejemplo: "La verdad la edición estuvo hermosa. Que buenas tomas, y Barcelona precioso :3" (Comentario al vídeo de Wismichu).

- Amor ( $n=85,8,5 \%)$ : El autor del comentario expresa palabras/sentimientos con connotación amorosa hacia el youtuber. Por ejemplo: "La más hermosa, talentosa, humilde, tierna! No sabes cuanto TE AMO. daría todo por abrazarte <3" (Comentario al vídeo de Dulceida).

- Pide al youtuber que desarrolle un contenido $(n=68,6,8 \%)$ : El autor del comentario solicita al youtuber que desarrolle un contenido o video sobre algún tema. Por ejemplo: "Isma, deberías hacer un video contando la alimentación que tuviste al volverte vegetariano y la transición de ser vegetariano a vegano. Me interesa mucho. Un saludo" (Comentario al vídeo de Wismichu).

2)Similitud con el youtuber: los seguidores destacan que se reconocen en el youtuber o en sus experiencias vitales ( $\mathrm{n}=99,9,9 \%)$. Por ejemplo:

"Me siento súper identificada contigo, respecto a tu respuesta sobre ser borde y respecto a tu respuesta de ser sensible, soy totalmente como tú" (Comentario al vídeo de Herrejón).

3)Identificación aspiracional: algunos usuarios también señalan el deseo de ser o parecerse al youtuber $(n=18,1,8 \%)$. Por ejemplo:

"Yo es que cada vez más fan...de verdad, ERES INCREIBLE, vaya ejemplazo a seguir :)" (Comentario al vídeo de Herrejón).

4)Entender al youtuber: algunos seguidores señalan que entienden y comparten los sentimientos del youtuber $(n=10,1 \%)$. Por ejemplo:

"Se te ve súper enérgico y feliz y yo no puedo estar más contenta por ello. gracias por el video, echaba de menos verte más seguido por aquí. y por cierto, eres guapísimo empieza a creertelo ya. ily\#\#" (Comentario al vídeo de Celopan).

\subsection{Motivos de agrado}

A continuación, se presentan los rasgos del youtuber que más se han citado en los comentarios de los seguidores:

1)Humor/Diversión: los seguidores valoran que el youtuber les divierta y tenga gracia $(n=100,10 \%)$. Por ejemplo:

"Eres muy graciosa me hiciste reír varias veces \#\#\# jajajaja quisiera poner los minutos en qué me hiciste reír pero estaría muy largo mi comentario \#\# POSDATA: es el primer video que veo tuyo y ya me suscribi;) ME HICISTE EL DÍA" (Comentario al vídeo de Yellow Mellow). 
2)Personalidad: los seguidores señalan aspectos prosociales o positivos de la personalidad del youtuber, como su esfuerzo para lograr sus sueños o su altruismo ( $n=96,9,6 \%)$. Por ejemplo:

"Eres la mejor, siempre te preocupas por toda la gente que te sigue \#\# Te queremos \#\#" (Comentario al vídeo de Paula Gonu).

3)Atractivo físico: los seguidores también valoran el atractivo físico o cuestiones relativas al aspecto físico del youtuber (vestimenta, peinado, etc.) $(n=76,7,6 \%)$. Por ejemplo:

"Me fascina tu color de pelo y tus labios dios \#\#\#" (Comentario al vídeo de Herrejón).

4) Inteligencia: otro rasgo destacado en los comentarios es que el youtuber sea inteligente $(n=15,1,5 \%)$. Por ejemplo:

"23:14 eso es tener hambre de conocimiento. Leer de todo. Es lo más inteligente. No encerrarse en un solo tema y burlarse del otro. Más gente así, por favor" (Comentario al vídeo de Jaime Altozano).

5)Riqueza/Éxito: los usuarios han señalado, en menor medida, el éxito personal o profesional del youtuber o su riqueza $(n=5,0,5 \%)$. Por ejemplo:

"Preciosaaaa, te quedo increíble \# Saludos y sigue asi de exitosa\#" (Comentario al vídeo de Dulceida).

\section{Discusión}

Este estudio responde a la escasez de investigaciones sobre el impacto de los influencers y, específicamente, de los youtubers, en sus seguidores (Rego y Romero-Rodríguez, 2016; Aran-Ramspott et al., 2018; Elorriaga y Monge, 2018; Tolbert y Drogos, 2019). Así, los resultados aquí presentados permiten aproximarse al perfil de estos nuevos referentes desde la mirada de sus folllowers, lo que aporta un conocimiento novedoso a esta área de investigación.

Los estudios previos han puesto de relieve la capacidad socializadora del medio televisivo y sus personajes (Livingstone, 1998; Hoffner y Buchanan, 2005; Igartua, 2010; Korres y Elexpuru, 2016), así resulta relevante ampliar este nicho de investigación hacia los nuevos medios y referentes mediáticos. Hoy en día, las redes sociales son herramientas de entretenimiento, comunicación y socialización en el día a día, especialmente, de las generaciones millennials y centennials (AIMC, 2018; Kádeková y Holiencinová, 2018; Eurostat, 2020), destacando entre ellas YouTube. De este modo, los youtubers forman parte de la vida de las generaciones más jóvenes, influyendo en el desarrollo de su identidad, valores y actitudes (Aran-Ramspott et al., 2018; Fernández-de-Arroyabe-Olaortua et al., 2018). Por ello, resulta importante desarrollar investigaciones como la presentada, donde el análisis realizado sobre los comentarios e interacciones de los seguidores de los youtubers españoles de éxito, ha permitido ahondar en la relación que establecen con los mismos en términos de comentario y tema, identificación y motivos de agrado. 
En primer lugar, en cuanto al tema y la posición de los comentarios estudiados, se ha observado que, principalmente, los comentarios se refieren al tema del vídeo o al youtuber, posicionándose la mayoría a favor de su contenido, lo que es coherente con la comunidad generada en torno a los youtubers, que, en general, comparte intereses y opiniones (Regueira et al., 2020). También se ha observado que algunos seguidores hacen referencia a otros contenidos de la red o a otros youtubers o influencers, lo cual concuerda con el uso que hacen los usuarios, especialmente, los jóvenes, de las redes sociales: seguir a influencers (IAB Spain, 2018). Un aspecto por destacar es el uso de emoticonos y/o emojis en los comentarios, que es una característica en las interacciones digitales y "se han convertido en una nueva forma de comunicación que permite la proyección de nuestros sentimientos, emociones, estados de ánimo, intereses o percepciones particulares de las cosas" en las plataformas y redes virtuales (Montenegro y Hermenegildo, 2018, p. 38). El éxito de los emoticonos y/o emojis puede deberse a que facilitan la economía lingüística y la expresividad, y, a su vez, dado su uso relativamente consensuado en la comunidad de usuarios, favorecen la claridad de la comunicación (Cantamutto y Vela, 2019).

En segundo lugar, la identificación con los youtubers aparece como una categoría relevante en el análisis de los comentarios de los seguidores, coincidiendo con estudios previos relativos a la identificación con los personajes de ficción televisiva (Cohen, 2001; Eyal y Rubin, 2003; Hoffner y Buchanan, 2005; Moyer-Gusé, 2008; Korres y Elexpuru, 2016), y, por tanto, es un aspecto a considerar a la hora de abordar la relación que establecen con estos referentes mediáticos. Se ha comprobado que este proceso puede producirse por distintas razones, destacando la interacción parasocial, especialmente, para felicitar al youtuber por el trabajo realizado, mostrar palabras/sentimientos con connotación amorosa hacia este, y/o pedirle que desarrolle un nuevo contenido o video sobre algún tema. El hecho de que, comparado con los personajes ficticios de los medios tradicionales, los youtubers sean personas reales, favorece la vinculación de los seguidores (Tolbert y Drogos, 2019), que los perciben como personas cercanas y accesibles (Bonaga y Turiel, 2016; Regueira et al., 2020). De este modo, aunque la interacción parasocial también se da con relación a los personajes televisivos (Livingstone, 1998; Eyal y Rubin, 2003; Korres y Elexpuru, 2016), parece aumentar con los youtubers, lo que puede relacionarse con las facilidades de interacción que ofrece la plataforma YouTube respecto a los medios tradicionales, ya que en esta red social la audiencia no solo es espectadora, sino que puede participar activamente e interactuar tanto con el youtuber como son otros followers (De la Fuente, Lacasa y Martínez-Borda, 2019; Scolari y Fraticelli, 2016). Tras la interacción parasocial, destaca también la similitud con el youtuber, que se muestra como un aspecto relevante a la hora de fortalecer el vínculo, tanto con este tipo de influencers (Aran-Ramspott et al., 2018; Tolbert y Drogos, 2019) como con los personajes televisivos (Eyal y Rubin, 2003; Hoffner y Buchanan, 2005; Moyer-Gusé, 2008; Korres y Elexpuru, 2016). En menor medida, los seguidores hacen referencia a la identificación aspiracional y a entender al youtuber, cuya importancia ha sido destacada también por otros estudios, tanto en relación con los nuevos referentes mediáticos (Tolbert y Drogos, 2019) como en el ámbito de los medios tradicionales (Cohen, 2001; Hoffner y Buchanan, 2005; Moyer-Gusé, 2008; Korres y Elexpuru, 2016). Así, se concluye que, del mismo modo 
que los personajes televisivos, los youtubers, a través de mecanismos de identificación, pueden actuar como modelos de referencia y líderes de opinión (Aran-Ramspott et al., 2018; Fernández-de-Arroyabe-Olaortua et al., 2018), en mayor medida, incluso, que las celebridades tradicionales (Defy Media, 2016).

En tercer lugar, entre los rasgos del youtuber que más valoran los seguidores, se encuentran su humor y su personalidad, en línea con estudios previos tanto sobre los youtubers (Bonaga y Turiel, 2016; Aran-Ramspott et al., 2018; Tolbert y Drogos, 2019) como sobre los personajes de ficción televisiva (Medrano et al., 2010; Medrano y Martínez de Morentín, 2012; Bermejo, 2012; Korres y Elexpuru, 2016). Otro elemento que valoran los followers en sus comentarios es el atractivo físico del youtuber, en coincidencia con estudios previos tanto sobre este tipo de influencers (Aran-Ramspott et al., 2018) como sobre personajes televisivos (Ruiz, Conde y Torres, 2005; Korres y Elexpuru, 2016), aunque difiere de otros estudios que señalan que este rasgo no es importante a la hora de sentir atracción hacia los personajes de ficción televisiva (Medrano et al., 2010; Medrano \& Martínez de Morentín, 2012). Así, se concluye que, aunque este aspecto no emerge como el más relevante, no se debe desestimar su impacto en la construcción de la identidad de los más jóvenes. En menor medida, los seguidores hacen referencia a la inteligencia y a la riqueza/éxito del youtuber, en coherencia con la importancia que adquiere la inteligencia de los youtubers para la audiencia (Tolbert y Drogos, 2019), así como la inteligencia y la riqueza/ éxito de los personajes televisivos (Korres y Elexpuru, 2016). Sin embargo, en general, estas dos últimas categorías no han sido recogidas en trabajos previos, por lo que resulta oportuno continuar profundizando en la importancia que adquieren para los seguidores de los youtubers.

Entre las limitaciones de este trabajo cabe destacar que el estudio de los comentarios de la audiencia no permite ahondar en los resultados obtenidos a través de una interacción con los seguidores. También se debe considerar que, los perfiles de las redes sociales no permiten identificar de manera explícita el género, el origen o la edad de los followers, lo cual limita el análisis y la interpretación de los resultados en relación con estas variables.

Por este motivo, en futuras investigaciones, se propone generar una práctica similar con los youtubers favoritos de los adolescentes y los jóvenes, mediante focus groups, para conocer los perfiles de sus youtubers favoritos e identificar cuáles son los rasgos que más destacan en sus youtubers favoritos, así como los valores que perciben de los mismos, con el fin de seguir avanzando en el conocimiento sobre el impacto que tienen estos nuevos referentes en el proceso de desarrollo de la identidad adolescente. A partir de este conocimiento se podrá favorecer el desarrollo de propuestas educativas orientadas a promover la alfabetización mediática y la lectura reflexiva y crítica sobre los discursos de los youtubers, especialmente, en relación con los valores que se perciben y los aprendizajes que se adquieren a través de estos nuevos referentes mediáticos. 


\section{Referencias}

AIMC (2018). 20 Navegantes en la Red, 2018. Madrid: AIMC.

Aran-Ramspott, S., Fedele, M. y Tarragó, A. (2018). Funciones sociales de los Youtubers y su influencia en la preadolescencia. Comunicar, 57, 71-80. https://doi.org/10.3916/C57-2018-07

Bermejo, J. (2012). Los personajes y las series de ficción en la vida de los y las jóvenes. Revista de Estudios de Juventud, 96, 31-49. Recuperado de https://bit.ly/3bSjBwX

Bonaga, C. y Turiel, H. (2016). Mamá, iquiero ser youtuber! Madrid: Temas de Hoy.

Buckingham, D. (2008). Youth, Identity and Digital Media. Cambridge, MA: The MIT Press.

Callejo, J. y Gutiérrez, J. (2014). La comunicación de los jóvenes adolescentes en las redes sociales. Revista interdisciplinar de estudios de comunicación y ciencias sociales, 19, 11-29. Recuperado de http://hdl.handle.net/10637/6618

Cantamutto, L. y Vela, C. (2019). Emojis frecuentes en las interacciones por WhatsApp: estudio comparativo entre dos variedades de español (Argentina y España). Círculo de Lingüística Aplicada a la Comunicación, 77, 171-186. http://dx.doi.org/10.5209/CLAC.63282

Cohen, J. (2001). Defining identification: a theoretical look at the identification audiences with media characters. Mass Communication and Society, 4(3), 245-265. https://doi.org/10.1207/S1532782 5MCS0403_01

Cover, R. (2012). Performing and undoing identity online: Social networking, identity theories and the incompatibility of online profiles and friendship regimes. Convergence: The International Journal of Research into New Media Technologies, 18(2), 177-193. https://doi.org/10.1177/13548 $\underline{56511433684}$

Defy Media (2016). Youth Video Diet: Acumen Report. Defy Media. Recuperado de https://bit.ly/3ccV $\underline{\mathrm{Obb}}$

De la Fuente, J., Lacasa, P. y Martínez-Borda, R. (2019). Adolescentes, redes sociales y universos transmedia: la alfabetización mediática en contextos participativos. Revista Latina de Comunicación Social, 74, 172-196. https://doi.org/10.4185/RLCS-2019-1326

De Veirman, M., Cauberghe, V. y Hudders, L. (2016). Marketing through Instagram influencers: impact of number of followers and product divergence on brand attitude. International Journal of Advertising, 36(5), 798-828. https://doi.org/10.1080/02650487.2017.1348035

Díaz-Aguado, Ma J. y Falcón, L. (2013). La identidad del estudiante adolescente en los relatos audiovisuales de ficción como tarea de alfabetización mediática. Revista de Estudios de Juventud, 101, 103-121. Recuperado de https://bit.ly/3mz2dSP

Elorriaga, A. y Monge, S. (2018). La profesionalización de los YouTubers: el caso de Verdeliss y las marcas. Revista Latina de Comunicación Social, 73, 37-54. https://doi.org/10.4185/RLCS-2018-1 244

Escobar, M. y Román, H. (2011). La presentación del yo en el ciberespacio: un análisis de las autodefiniciones personales en blogs y redes sociales. Revista de Psicología Social, 26(2), 207-222. doi: https://doi.org/10.1174/021347411795448947

Eurostat (2020). Being Young in Europe today. Recuperado de http://bit.ly/39wtjE2

Eyal, K. y Rubin, A. M. (2003). Viewer aggression and homophily, identification and parasocial relationships with television characters. Journal of Broadcasting and Electronic Media, 47, 77-98. https://doi.org/10.1207/s15506878jobem4701 5 
Fedele, M., Aran-Ramspott, S., Elexpuru, I. y Korres, O. (en prensa). Construcción de las identidades, valores y estereotipos juveniles en los social media: I@s influencers y las audiencias Millennial y Centennial. Madrid: Editorial Dykinson.

Fernández-de-Arroyabe-Olaortua, A., Lazkano-Arrillaga, I. y Eguskiza-Sesumaga, L. (2018). Nativos digitales: Consumo, creación y difusión de contenidos audiovisuales online. Comunicar, 57(26), 61-69. https://doi.org/10.3916/C57-2018-06

Hoffner, C. y Buchanan, M. (2005). Young adults wishful identification with television characters: the role of perceived similarity and character attributes. Media Psychology, 7, 325-351. https:/ /doi.org/10.1207/S1532785XMEP0704_2

IAB Spain (2018). Estudio anual de redes sociales. Madrid: IAB. Recuperado de https://bit.ly/3fMTOb3 Igartua, J.J. (2010). Identification with characters and narrative persuasion through fictional feature films. Communications: The European Journal of Communication Research, 35(4), pp. 347-373. $\underline{\mathrm{h}}$ ttps://doi.org/10.1515/comm.2010.019

Kádeková, Z. y Holienčinová, M. (2018). Influencer marketing as a modern phenomenon creating a new frontier of virtual opportunities. Communication Today, 9(2), 90-105. Recuperado de ht tps://bit.ly/2NfmPSe

Korres, O. y Elexpuru, I. (2016). Las preferencias de los adolescentes sobre los personajes televisivos de ficción seriada. Trípodos, 38, 141-159. Recuperado de http://bit.ly/39yO9D4

Larrañaga, J. y Ruiz, A.N. (2009). El modelo de negocio de YouTube. Icono 14, 12, 109-131. Recuperado de https://bit.ly/2S8GIxE

Livingstone, S. (1998). Making Sense of Television. The Psychology of Audience Interpretation (2 ed.). London: Routledge.

Medrano, C., Cortés, A., Aierbe, A. y Orejudo, S. (2010). Los programas y características de los personajes preferidos en el visionado de televisión: diferencias evolutivas y de sexo. Cultura y Educación, 22(1), 3-20. https://doi.org/10.1174/113564010790935196

Medrano, C. y Martínez de Morentin, J.I. (2012). Socialización y televisión: Perfiles de adolescentes en un estudio transcultural. International Journal of Developmental and Educational Psychology, 1(1), 675-682. Recuperado de https://bit.ly/332vlZS

Montenegro, D. y Hermenegildo, E. (2018) Características del uso de emojis en la comunicación por el chat de WhatsApp. Hamut 'ay, 5(1), 36-52. http://dx.doi.org/10.21503/hamu.v5i1.1519

Morduchowicz, R., Marcon, A., Sylvestre, V. y Ballestrini, F. (2010). Los adolescentes y las redes sociales. Escuelas y Medios. Ministerio de Educación de la Nación de Argentina.

Morgan, B. y Kristjánsson, K. (2017). Parents and Social Media. Adolescents' perceptions of parental responses to morally salient social media scenarios. Birmingham: University of Birmingham and The Jubilee Center for Character and Virtues

Moyer-Gusé, E. (2008). Toward a theory of entertainment persuasion: Explaining the persuasive effects of entertainment-education messages. Communication Theory, 18(3), 407- 425. https:// doi.org/10.1111/j.1468-2885.2008.00328.x

Rego, S. y Romero-Rodríguez, L. (2016). Representación discursiva y lenguaje de los 'youtubers' españoles: Estudio de caso de los 'gamers' más populares. Index.comunicación, 6(1), 197-224. Recuperado de http://bit.ly/3oKSljM

Regueira, U., Alonso-Ferreiro, A. y Da-Vila, S. (2020). Women on YouTube: Representation and participation through the Web Scraping technique. Comunicar, 28(63), 31-40. https://doi.org/ 10.3916/C63-2020-03 
Renés, P., Gozálvez, V. y Berlanga, I. (2020). YouTube e influencers en la infancia. Análisis de contenidos y propuestas educativas. Icono 14, 18(2), 269-295. https://doi.org/10.7195/ri14.v1 $\underline{8 i 2.1455}$

Ruiz, C., Conde, E. y Torres, E. (2005). Importance of facial physical attractiveness of audiovisual models in descriptions and preferences of children and adolescents. Perceptual and Motor Skills, 101, 229-243. https://doi.org/10.2466/pms.101.1.229-243

Sabich, M.A. y Steinberg, L. (2017). Discursividad youtuber: afecto, narrativas y estrategias de socialización en comunidades de Internet. Revista Mediterránea de Comunicación, 8(2), 171-188. http://dx.doi.org/10.14198/MEDCOM2017.8.2.12

Scolari, C. y Fraticelli, D. (2016). Nuevos sujetos mediáticos en el ecosistema de medios. V Congreso AsAECA, Buenos Aires.

Tolbert, A.N. y Drogos, K.L. (2019). Tweens' Wishful Identification and Parasocial Relationships with YouTubers. Frontiers in Psychology, 10, 1-15. https://doi.org/10.3389/fpsyg.2019.02781

Vizcaíno-Verdú, A., De Casas-Moreno, P. y Aguaded, I. (2019). Youtubers e instagrammers: Una revisión sistemática cuantitativa. En I. Aguaded, A. Vizcaíno-Verdú y Sandoval-Romero, Y. (Eds.). Competencia mediática y digital. Del acceso al empoderamiento (pp. 211-219). Huelva: Grupo Comunicar Ediciones.

Youtube (2021). Youtube para prensa. Recuperado de http://bit.ly/3qjTzla

Notas

1 Este trabajo presenta parte de los resultados de la investigación “Construcción de las identidades de género, valores y estereotipos juveniles en los social media: I@s influencers y las audiencias Millennial y Centennial", financiado por el Centro Reina Sofía (VI convocatoria de ayudas a la investigación sobre adolescencia y juventud). Del 01-03-2019 al 30-07-2020. 